\title{
ESTADIAMENTO DO CÂNCER DE MAMA DIAGNOSTICADO NO SISTEMA PÚBLICO DE SAÚDE DE SÃO CARLOS
}

\author{
THE STAGING OF BREAST CANCER DIAGNOSED \\ IN SÃO CARLOS PUBLIC HEALTH SYSTEM
}

Alexandre Henrique Macchetti

Pós-graduando. Departamento de Ginecologia e Obstetrícia. Faculdade de Medicina de Ribeirão Preto - USP. Médico Assistente. Serviço de Mastologia da Secretaria de Saúde de São Carlos, São Carlos - Brasil

Correspondência: Dr. Alexandre Henrique Macchetti - Rua Maria Jacinta, 241, sala 81. São Carlos - SP - Brasil - CEP: $13560-610$

Tel/Fax: 16-3376-3460 / e-mail: ahmacchetti@yahoo.com.br

Macchetti AH. Estadiamento do câncer de mama diagnosticado no Sistema Público de Saúde de São Carlos. Medicina (Ribeirão Preto) 2007; 40 (3): 394-402, jul./set.

RESUMO: Introdução: dados sobre o estadio do câncer no momento do diagnóstico são facilmente disponíveis para a maioria dos países desenvolvidos, mas para os países da América Latina estes são difíceis de serem obtidos. Dados do Instituto Nacional do Câncer referem $60 \%$ dos casos de câncer de mama em estadio avançado, no entanto o presente do Serviço de Mastologia da Secretaria de Saúde de São Carlos mostrou dados diversos que estimularam a avaliação da idade e do estadio do câncer de mama dos pacientes do Sistema Único de Saúde residentes na cidade de São Carlos e a comparação às estatísticas de outras instituições. Pacientes e Métodos: registros de 106 pacientes com diagnóstico de câncer de mama entre Março de 2000 e Dezembro de 2004 no Serviço de Mastologia da Secretaria de Saúde de São Carlos (SUS) foram revisados quanto ao estadio e a idade no momento do diagnóstico. Os casos de câncer de mama foram agrupados segundo os sistemas de estadiamento TNM e SEER, e a idade conforme valor acima ou abaixo da idade mediana do grupo de pacientes com o objetivo de correlacioná-los. Resultados: no sistema de estadiamento TNM os tumores in situ corresponderam a $6,6 \%$ de todos os casos, tumores no estadio I a $25,4 \%$, estadio II a $44,3 \%$, estadio III a $17,0 \%$ e estadio IV a $6,6 \%$. No sistema de estadiamento SEER os tumores in situ corresponderam a $6,6 \%$ de todos os casos, tumores locais a $62,2 \%$, tumores regionais a $24,5 \%$, e disseminados a $6,6 \%$. Houve mais casos em estadio inicial utilizando o sistema TNM $(76,3 \%)$ do que o sistema SEER (68,8\%). Houve associação do grupo de pacientes com menos de 56 anos com o estadio inicial ao diagnóstico com o sistema TNM, mas não com o sistema SEER. Conclusões: dados relevantes para o melhor entendimento da atenção ao câncer de mama são apresentados, permitindo melhora das intervenções ao nível da comunidade para detecção do câncer e também uma estratégia dirigida por dados na alocação de recursos para políticas, programas e serviços relacionados ao câncer.

Descritores: Neoplasias Mamárias. Estadiamento de Neoplasias. Epidemiologia dos Serviços de Saúde. 


\section{1- INTRODUÇÃO}

O câncer de mama, após o câncer de pele não melanoma, é a neoplasia mais comumente diagnosticada entre as mulheres brasileiras com uma estimativa de 48.930 casos novos em $2006^{1}$. As estatísticas têm demonstrado aumento significativo de incidência da doença (cerca de 1\% ao ano em muitos países), mas mortalidade estável por muitas décadas, o que pode demonstrar melhoria na detecção precoce e no tratamento ${ }^{1,2}$. O prognóstico destas pacientes está intimamente relacionado ao estadio em que os tumores são diagnosticados, logo a implantação de programas de detecção precoce do câncer de mama é fundamental $^{1}$. O objetivo dos programas de detecção precoce do câncer de mama é a detecção ainda em estadios não invasivos (carcinoma in situ), reduzindo a freqüência de desenvolvimento de câncer de mama invasivo, tanto quanto aumentando a identificação do câncer de mama em estadios iniciais (TNM estadio I) que possibilitam além de um tratamento com menor morbidade uma chance de cura maior que $90 \% \%^{2,3}$.

Embora os primeiros reflexos destes programas sobre a mortalidade levem ao menos 10 anos para serem notados, a efetividade destes pode ser avaliada indiretamente com a análise do estadio do câncer de mama no momento do diagnóstico ${ }^{2,3,4}$. Os sistemas de estadiamento mais freqüentemente empregados são o TNM da American Joint Committee on Cancer (AJCC) e o Summary Staging System do Instituto Nacional do Câncer dos Estados Unidos da América e, independente do sistema empregado, dados sobre o câncer de mama nas últimas décadas são facilmente disponibilizados pelos países desenvolvidos, enquanto para a maioria dos países em desenvolvimento, entre eles os latino-americanos, estes são muito mais difíceis de serem obtidos ${ }^{5,6,7}$.

O diagnóstico do câncer de mama em estadio inicial tem sido associado com muitas variáveis, entre elas a posição a sócio-econômica e o acesso à saúde oferecido à população $0^{8,9,10}$. Estudos norte-americanos verificaram que a baixa renda é um fator de risco para o diagnóstico da doença em estadio avançado e para o tratamento incompleto, e que as mulheres que têm o seu acesso à saúde realizado por programas governamentais, como Medicaid, têm seus tumores diagnosticados em um estadio mais avançado em relação às mulheres que utilizam prestadores de saúde contratados $^{9 / 14}$.
Os dados utilizados para expressar o padrão de estadiamento do câncer de mama na grande maioria dos artigos provêm de dados oficiais do Instituto $\mathrm{Na}$ cional do Câncer (INCA), e relata-se que ao redor de $60 \%$ dos tumores mamários são detectados em estadio avançado, mas os dados do Serviço de Mastologia da Secretaria de Saúde de São Carlos (SM São Carlos) não foram compatíveis com tal relato, pois se encontra a prevalência de casos em estadio inicial, o que mostra a relevância deste estudo ${ }^{1,7}$.

O principal objetivo do deste estudo foi verificar qual o padrão do estadiamento do câncer de mama no Serviço de Mastologia da Secretaria de Saúde de São Carlos, componente do hierarquizado Sistema Único de Saúde (SUS) neste município, de uma forma padronizada e que permita a comparação com os dados de outras instituições nacionais e internacionais. Os dados revelaram-se nacionalmente significantes, mas ainda aquém dos observados nas populações com programas de rastreamento mais efetivos, podem ser utilizados no planejamento de ações de saúde e como referência para o acompanhamento da efetividade dos programas implantados.

\section{2- PACIENTES E MÉTODOS}

Um estudo descritivo dos registros de 106 pacientes com câncer de mama diagnosticado entre 1 de Março de 2000 e Dezembro de 2004 no Serviço de Mastologia da Secretaria de Saúde de São Carlos (SUS) foi realizado. Os prontuários foram revisados quanto ao estadio do câncer de mama e a idade da paciente no momento do diagnóstico. Todos os dados coletados neste estudo referiram-se àqueles das pacientes residentes no município de São Carlos, para o qual este serviço é a referência para o diagnóstico e tratamento cirúrgico do câncer de mama, logo os dados de uma parcela considerável de pacientes residentes em municípios de nossa região, ainda que em seguimento neste serviço, não foram computados nesta análise, pois não seria possível assegurar que todos os casos fossem a nós encaminhados gerando uma amostra que não correspondesse à nossa realidade.

Todas as pacientes tiveram os seus tumores estadiados pelos dois principais sistemas aplicados na literatura revisada: o TNM da AJCC, o mais empregado em nosso país, e o SEER Summary Staging System do Programa Surveillance, Epidemiology, and 
End Results (SEER) do Instituto Nacional do Câncer dos Estados Unidos da América. A Associação Norte-Americana de Centros de Registro de Câncer é quem provê as instruções de codificação de todos os casos diagnosticados a partir de 2001, e devido a este fato, apesar de sua pequena disseminação em nosso meio, também empregamos o sistema de estadiamento por eles utilizado, o SEER, como forma de tornarmos os dados do SM São Carlos comparáveis. O intercâmbio dos dados finais apresentados por estes dois sistemas de estadiamento não é totalmente possível após serem estabelecidos, pois há grandes diferenças nos seus critérios, e isto estimulou a disponibilização dos dados em ambos os sistemas ${ }^{15,16}$.

$\mathrm{Na}$ literatura revisada acerca o estadiamento do câncer de mama os estadios 0, I e II do sistema TNM da AJCC são considerados componentes do grupo câncer de mama em estadio inicial, e os tumores em estadio III e IV como componentes do grupo câncer de mama em estadio tardio. Os estadios 0 e I compõem o grupo de tumores mamários com baixo estadio e os tumores com estadio IIa ou superior compõem o grupo com alto estadio.

O sistema SEER basicamente organiza os estadios conforme a disseminação do tumor além do seu ponto de origem. No sistema SEER os tumores in situ são definidos como aqueles que não penetraram a membrana basal do tecido nem se estenderam além do epitélio, os tumores locais referem-se à neoplasia maligna e invasiva que está confinada totalmente ao orgão de origem, os tumores regionais referem-se à neoplasia maligna e invasiva que se estendeu além do orgão de origem, acometendo os tecidos ao redor, envolvendo os linfonodos, ou ambos, e finalmente os tumores disseminados que se referem aqueles que disseminaram às partes remotas do corpo a partir do sítio primário por extensão direta ou por metástases. $\mathrm{O}$ grupo "câncer de mama em estadio inicial" é composto pelos tumores in situ e locais apenas.

A média de idade das pacientes foi de 57,3 anos e a mediana de 56 anos (26 - 89 anos), logo grupos de idade "abaixo de 56 anos" e "56 anos e acima" foram utilizados para analisar uma associação entre grupo etário e estadio do câncer de mama. Uma comparação do diagnóstico do câncer de mama em estadio inicial entre os períodos de 2000-2002 e 2003-2004 foi também realizada. O programa de análise estatística GraphPad Prism (versão 3.00, GraphPad Incorporated, San Diego, USA) foi utiliza- do para analisar todos os dados. Os dados foram comparados pele teste Qui-quadrado, e um valor de $p$ menor que 0,05 foi considerado como estatisticamente significante.

\section{3- RESULTADOS}

O estadio dos tumores conformes os sistemas TNM e SEER foram avaliados em todos os casos. No sistema TNM as pacientes com tumores estadio 0 ou in situ corresponderam a $6,6 \%$ de todos os casos, com estadio I a $25,4 \%$, com estadio II a $44,3 \%$, com estadio III a $17,0 \%$ e com estadio IV a $6,6 \%$. Considerando todos os casos o estadio clínico T2N0M0 foi o mais prevalente constituindo $33 \%$ das pacientes $(n=35)$. A Tabela I mostra as pacientes agrupadas de acordo com o estadio TNM para cada ano do período estudado. Durante o período 2000-2004, o percentual médio de tumores em estadio inicial foi de $76,3 \%$.

No sistema SEER as pacientes com tumores in situ corresponderam a $6,6 \%$ de todos os casos, os tumores considerados como localizados a $62,2 \%$, os tumores considerados regionais a $24,5 \%$, e os tumores disseminados a $6,6 \%$. A Tabela II mostra as pacientes agrupadas de acordo com o estadio SEER para cada ano do período estudado. Durante o período 2000-2004, o percentual médio de tumores em estadio inicial foi de $68,8 \%$ e a relação estadio inicial/ tardio foi de 2,21.

Casos de câncer de mama em estadio inicial foram mais freqüentes quando utilizado o sistema TNM (76,3\%) em comparação ao sistema $\operatorname{SEER}(68,8 \%)$, como esperado pelas diferenças nos critérios utilizados para o agrupamento dos tumores em estadio inicial e tardio.

Houve a associação entre o grupo etário "abaixo de 56 anos" com câncer de mama em estadio inicial no sistema TNM ( $p=0,011)$, mas não no sistema SEER, provavelmente pelas diferenças nos critérios utilizados para o agrupamento dos tumores em estadio inicial e tardio (Tabela III). Esta associação não foi observada quando a comparação foi realizada entre tumores classificados como de baixo estadio (TNM estadio 0 e I). Não houve mudança no padrão de distribuição dos casos dentre os grupos de estadios em ambos os sistemas de estadiamento comparando os períodos 2000-2002 a 2003-2004. 
Tabela I - Distribuição percentual de tumores conforme estadio TNM por ano e comparação entre agrupamento de estadios entre os períodos de 2000-2002 e 2003-2004: Serviço de Mastologia da Secretaria de Saúde de São Carlos (SUS), 2000-2004

\begin{tabular}{|c|c|c|c|c|c|c|c|c|c|c|c|}
\hline \multirow{5}{*}{ Ano-Período } & \multicolumn{11}{|c|}{ Estadio TNM } \\
\hline & \multirow{3}{*}{ Total } & \multicolumn{6}{|c|}{ Estadio inicial ${ }^{\mathrm{a}, \mathrm{c}}$} & \multirow{2}{*}{\multicolumn{4}{|c|}{$\begin{array}{l}\text { Estadio tardio } \\
\text { adio alto }\end{array}$}} \\
\hline & & \multicolumn{4}{|c|}{ Estadio baixo ${ }^{a, c}$} & \multirow{2}{*}{\multicolumn{2}{|c|}{ II }} & & & & \\
\hline & & \multicolumn{2}{|c|}{0} & \multicolumn{2}{|c|}{ I } & & & \multicolumn{2}{|c|}{ III } & \multicolumn{2}{|c|}{ IV } \\
\hline & $\mathrm{n}$ & $\%$ & $\mathrm{~N}$ & $\%$ & $\mathrm{~N}$ & $\%$ & $\mathrm{~N}$ & $\%$ & $\mathrm{~N}$ & $\%$ & $\mathrm{~N}$ \\
\hline 2000 & 15 & 0 & 0 & 13,3 & 2 & 53,3 & 8 & 26,6 & 4 & 6,6 & 1 \\
\hline 2001 & 22 & 13,6 & 3 & 22,7 & 5 & 40,9 & 9 & 9,0 & 2 & 13,6 & 3 \\
\hline 2002 & 25 & 8,0 & 2 & 24,0 & 6 & 52,0 & 13 & 12,0 & 3 & 4,0 & 1 \\
\hline 2003 & 16 & 6,2 & 1 & 43,7 & 7 & 37,5 & 6 & 6,2 & 1 & 6,2 & 1 \\
\hline 2004 & 28 & 3,5 & 1 & 25 & 7 & 39,2 & 11 & 28,5 & 8 & 3,5 & 1 \\
\hline $2000-2004$ & 106 & 6,6 & 7 & 25,4 & 27 & 44,3 & 47 & 17,0 & 18 & 6,6 & 7 \\
\hline
\end{tabular}

a - teste $\chi^{2}$ para comparação dos casos de câncer de mama com diagnóstico em estadio inicial/tardio e baixo/alto entre os períodos de 2000-2002 e 2003-2004.

$\mathrm{b}-\mathrm{p}<0,05$ para significância estatística

$\mathrm{c}-\mathrm{p}>0,05$

Tabela II - Distribuição percentual de tumores conforme estadio SEER por ano e comparação entre agrupamento de estadios inicial e tardio entre os períodos de 2000-2002 e 2003-2004: Serviço de Mastologia da Secretaria de Saúde de São Carlos (SUS), 2000-2004,

\begin{tabular}{|c|c|c|c|c|c|c|c|c|c|c|}
\hline \multirow{4}{*}{ Ano } & \multirow{4}{*}{$\begin{array}{c}\text { Total de } \\
\text { casos }\end{array}$} & \multirow{4}{*}{$\begin{array}{c}\text { Razão } \\
\text { inicial/tardio }\end{array}$} & \multicolumn{8}{|c|}{ Estadio SEER } \\
\hline & & & \multicolumn{4}{|c|}{ Estadio inicial $^{\mathrm{a}, \mathrm{c}}$} & \multicolumn{4}{|c|}{ Estadio tardio } \\
\hline & & & \multicolumn{2}{|c|}{ in situ } & \multicolumn{2}{|c|}{ Local } & \multicolumn{2}{|c|}{ Regional } & \multicolumn{2}{|c|}{ Disseminado } \\
\hline & & & $\%$ & $\mathrm{~N}$ & $\%$ & $\mathrm{~N}$ & $\%$ & $\mathrm{~N}$ & $\%$ & $\mathrm{~N}$ \\
\hline 2000 & 15 & 1,5 & 0 & 0 & 60,0 & 9 & 33,3 & 5 & 6,7 & 1 \\
\hline 2001 & 22 & 2,1 & 13,6 & 3 & 54,5 & 12 & 18,1 & 4 & 13,6 & 3 \\
\hline 2002 & 25 & 3,1 & 8,0 & 2 & 68,0 & 17 & 20,0 & 5 & 4,0 & 1 \\
\hline 2003 & 16 & 3,0 & 6,2 & 1 & 68,7 & 11 & 18,7 & 3 & 6,2 & 1 \\
\hline 2004 & 28 & 1,8 & 3,5 & 1 & 60,7 & 17 & 32,1 & 9 & 3,5 & 1 \\
\hline $2000-2004$ & 106 & 2,21 & 6,6 & 7 & 62,2 & 66 & 24,5 & 26 & 6,60 & 7 \\
\hline
\end{tabular}

a - teste $\chi 2$ para comparação dos casos de câncer de mama estadio inicial/tardio entre os períodos de 2000-2002 e 2003-2004.

$\mathrm{b}-\mathrm{p}<0,05$ para significância estatística

$\mathrm{c}-\mathrm{p}>0,05$ 
Tabela III - Distribuição percentual de tumores conforme estadio TNM por grupos etários: Serviço de Mastologia da Secretaria de Saúde de São Carlos (SUS), 2000-2004,

\section{Estadio TNM}

\begin{tabular}{|c|c|c|c|c|c|c|c|c|c|c|c|c|}
\hline \multirow{4}{*}{$\begin{array}{l}\text { Grupo } \\
\text { Etário }\end{array}$} & \multirow{2}{*}{\multicolumn{2}{|c|}{$\begin{array}{c}\text { Total de } \\
\text { casos }\end{array}$}} & \multicolumn{6}{|c|}{ Estadio Inicial $^{\mathrm{a}, \mathrm{d}}$} & \multicolumn{4}{|c|}{ Estadio Tardio } \\
\hline & & & \multicolumn{4}{|c|}{ Baixo estadio $^{\mathrm{a}, \mathrm{c}}$} & \multicolumn{6}{|c|}{ Alto estadio } \\
\hline & & & \multicolumn{2}{|c|}{0} & \multicolumn{2}{|c|}{ I } & \multicolumn{2}{|c|}{ II } & \multicolumn{2}{|c|}{ III } & \multicolumn{2}{|c|}{ IV } \\
\hline & $\%$ & $\mathrm{~N}$ & $\%$ & $\mathrm{~N}$ & $\%$ & $\mathrm{~N}$ & $\%$ & $\mathrm{~N}$ & $\%$ & $\mathrm{~N}$ & $\%$ & $\mathrm{~N}$ \\
\hline Abaixo de 56 anos & 50 & 53 & 5,6 & 3 & 32,0 & 17 & 49,0 & 26 & 11,3 & 6 & 1,8 & 1 \\
\hline 56 anos e acima & 50 & 53 & 7,5 & 4 & 18,8 & 10 & 39,6 & 21 & 22,6 & 12 & 11,3 & 6 \\
\hline Total & 100 & 106 & 6,6 & 7 & 25,4 & 27 & 44,3 & 47 & 16,7 & 18 & 6,6 & 7 \\
\hline
\end{tabular}

a - teste $\chi 2$ para comparação dos casos de câncer de mama estadio inicial e baixo entre os grupos etários.

$\mathrm{b}-\mathrm{p}<0,05$ para significância estatística

$\mathrm{c}-\mathrm{p}>0,05$

$\mathrm{d}-\mathrm{p}=0,011$

Na Figura 1, os dados do SM São Carlos são comparados aos dados de serviços de referência nacionais para tratamento oncológico como o Hospital de Câncer de Barretos, a Unidade de Mastologia do Hospital de Base do Distrito Federal, ao Instituto Nacional do Câncer, e dados epidemiológicos de alguns estados norte-americanos ${ }^{17 / 23}$.

\section{4- DISCUSSÃO}

Estudos da efetividade do rastreamento mamográfico no diagnóstico do câncer de mama em estadio inicial praticamente inexistem no Brasil, entretanto podemos correlacionar o padrão de distribuição dos casos de câncer de mama estadiados por sistemas padronizados e o acesso ao rastreamento mamográfico, e obter indiretamente a efetividade dos programas de detecção precoce do câncer de mama implantados ${ }^{17 / 23}$.

No Brasil, os estudos de estadiamento do câncer de mama são realizados com séries de pacientes em hospitais públicos ou filantrópicos de referência à população geral, o que pode não refletir o real padrão de distribuição dos estadios do câncer de mama no SUS, uma vez que estes hospitais são referências para uma região muito ampla e que se sobrepõe a de outros serviços ${ }^{17,18,23,24}$. Considerando que o SM São Carlos é qualificado como a unidade de saúde de re- ferência para o diagnóstico e tratamento cirúrgico do câncer de mama de todos os casos do SUS no município de São Carlos no período deste estudo, podemos qualificar a casuística do SM São Carlos como representativa daquilo que ocorreu quanto ao diagnóstico do câncer de mama na população que foi assistida pelo SUS no período do estudo.

O percentual de diagnósticos feitos em estadios iniciais pelo sistema TNM, 75,4\%, é alto em relação aos poucos dados disponíveis no Brasil, obtidos de resumos de trabalhos apresentado no último Congresso Brasileiro de Mastologia em novembro de 2005 no Rio de Janeiro, o que demonstra a relevância dos dados do SM São Carlos, como discutido adiante, pode estar relacionado à algumas características ímpares do município de São $\operatorname{Carlos}^{17,18,23}$. O percentual de casos em estadio inicial pelo sistema SEER, $68,8 \%$, é comparável aos dados de alguns estados norte-americanos, entretanto o percentual de casos de tumores in situ é relativamente baixo ${ }^{19 / 22}$. Nos estados norteamericanos de Minnesota e Michigan os percentuais de tumores in situ correspondem, respectivamente, a $16,3 \%$ e $20,1 \%$ de todos os casos, enquanto a casuística do SM São Carlos mostra apenas $6,6 \%{ }^{19,20}$. No estado norte-americano de Rhode Island, um exemplo de população com bom programa de rastreamento, o percentual de tumores em estadio I foi de $41 \%$ de todos os tumores invasivos vs. $27 \%$ no SM São 


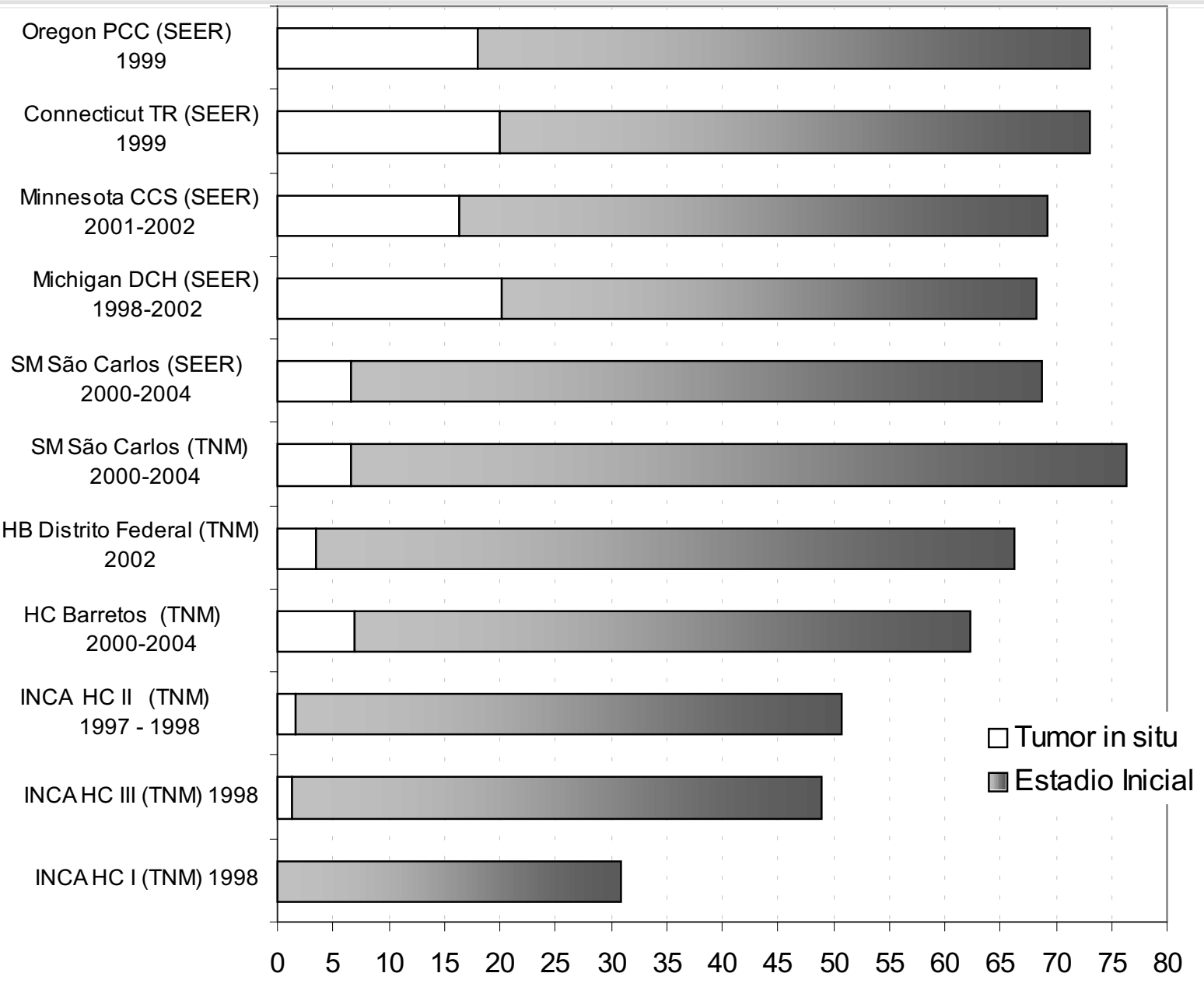

Figura 1: a- o sistema de estadiamento aparece entre parênteses antes do período dos dados.

b - barras brancas indicam o percentual de pacientes com câncer de mama in situ; as barras cinzas representam as mulheres com câncer de mama em estadio inicial, que compreendem os tumores in situ.

c - abreviaturas: Michigan DCH - Michigan Department of Community Health; Minnesota CSS - Minnesota Cancer Surveillance System; Oregon PCC - Oregon Partnership for Cancer Control; Connecticut TR - Connecticut Tumor Registry; HC Barretos Hospital de Câncer de Barretos; HBDF - Hospital de Base do Distrito Federal; INCA HC I - Hospital I do Instituto Nacional do Câncer; INCA HC II - Hospital II do Instituto Nacional do Câncer; INCA HC III - Hospital III do Instituto Nacional do Câncer; SM São Carlos - Serviço de Mastologia da Secretaria de Saúde de São Carlos.

Carlos ${ }^{25}$. Ainda que sejam observados dados relevantes quanto ao diagnóstico do câncer de mama em estadio inicial, infelizmente, apenas $22,1 \%$ de todos os tumores no SM São Carlos foram classificados como in situ ou tinham menos que 2 centímetros, ou seja, a menor parte dos casos estão sendo diagnosticados de forma subclínica, por meio do rastreamento mamográfico, e a maioria das pacientes ainda que sejam encaminhadas em estadio inicial têm o tumor mamário diagnosticado pelo exame clínico do médico ou autoexame. Considerando que apenas 10 a $15 \%$ de todos os casos de câncer de mama não são detectados pela mamografia, pode-se presumir que a abordagem da nossa população pelo programa de rastreamento não está tendo a efetividade que foi alcançada em outras populações.

Dados do SM São Carlos mostram a associação entre diagnóstico do câncer de mama em estadio tardio e o grupo etário acima de 56 anos, podendo indicar, neste grupo mais especificamente, que as campanhas públicas de incentivo ao auto-exame e mamografia anual não estão tendo a penetração desejada, pois estudos prévios mostram que o câncer de mama tende a ser encontrado em estadios mais avançados 
nas pacientes idosas, mas o rastreamento mamográfico regular é capaz de eliminar as disparidades quanto ao tamanho e estadio do câncer de mama entre os grupos etários ${ }^{8,9,11,26,27}$. Esta associação não foi encontrada com a utilização do sistema SEER, isto pode decorrer de dificuldades que surgem quando comparamos a distribuição dos casos de câncer de mama com relação ao estadio entre os dois diferentes sistemas de estadiamento utilizados, o TNM e o SEER do Instituto Nacional do Câncer dos Estados Unidos da América.

Um tumor mamário designado como in situ pelo sistema SEER corresponde ao estadio 0 do sistema TNM, mas um tumor considerado como localizado pelo primeiro sistema pode ser classificado como estadio I ou II dependendo do tamanho do tumor, ainda, um tumor caracterizado como regional pode ser estadiado em II ou III, dependendo do tamanho, extensão à parede torácica ou pele, ou acometimento de linfonodos regionais por disseminação neoplásica ${ }^{15,16}$. Observase que um tumor estadio II pelo sistema TNM pode ser classificado como local ou regional pelo sistema do SEER, indicando que a conversão dos estadios não é possível a menos que se disponha de todas as características do tumor no momento do diagnóstico ${ }^{15,16}$. Um tumor considerado disseminado pode ser classificado como estadio TNM III ou IV, dependendo da disseminação a linfonodos secundários ou metástases a distância ${ }^{15,16}$.

A comparação entre os períodos 2000-2002 e 2003-2004 não mostrou mudança no padrão de distribuição dos estadios, no entanto o tamanho da população e o curto espaço de tempo do estudo não permitem que a efetividade do programa de detecção precoce do câncer de mama, composto por campanhas publicitárias de incentivo ao auto-exame e conscientização da necessidade de realização de mamografias periódicas, seja questionada. Dados da Fundação Oncocentro de São Paulo mostram que a mortalidade por câncer de mama na população feminina paulista apresentou tendência de aumento passando de 11,7 por 100.000 mulheres no biênio $1987-88$ para $12,9 \mathrm{em}$ 1997-9828.

A variabilidade geográfica do diagnóstico do câncer de mama observada no Brasil muito provavelmente está relacionada às condições de vida e à capacidade de diagnósticos dos serviços de saúde disponibilizados de forma irregular pelo país ${ }^{24}$. No Brasil, o rastreamento mamográfico é irregular visto a concentração dos mamógrafos nas regiões sul e sudeste e a dependência de grande parte da população do SUS para o acesso à saúde ${ }^{24}$. O estado de São Paulo estimou para 2005 uma incidência de 103,4 casos novos de câncer de mama para 100.000 mulheres, e no biênio 2001-2002 uma mortalidade de 14,7 mulheres foi observada ${ }^{28}$. O Município de São Carlos pertence à DIR de Araraquara que no biênio 2001-2002 apresentou uma mortalidade de 12 mulheres/100.000 habitantes $^{28}$. O município conta com 3 mamógrafos para uma população de 82.958 mulheres com mais de 10 anos de idade, uma boa taxa de 2,48 unidades de diagnóstico mamográfico por 10.000 mulheres, sendo ainda disponibilizados aos pacientes a localização estereotáxica pré-operatória de lesões mamárias e também técnicas minimamente invasivas como a punção biópsia aspirativa e core biopsy guiada por ultrasonografia ${ }^{29,30}$. Técnicas modernas da medicina nuclear como a localização de lesões mamárias não palpáveis e do linfonodo sentinela pela marcação com rádio isótopos também foram recentemente incorporadas ao arsenal propedêutico disponibilizado aos usuários do SUS. Assim, pode-se afirmar que amplo acesso a um programa de rastreamento mamográfico e a disponibilização de técnicas minimamente invasivas de investigação associadas ao tratamento com baixa da morbidade para os casos em estadio inicial podem estar relacionados ao alto percentual de diagnóstico do câncer mamário em estadio inicial ${ }^{29,30}$.

O Índice de Desenvolvimento Humano (IDH) é composto pela conquistas de um município quanto às três dimensões básicas do desenvolvimento humano: uma vida longa e saudável, mensurada pela expectativa de vida ao nascimento; o conhecimento, mensurado pela taxa de instrução dos adultos e taxa de escolas primárias, secundárias e terciárias; e um padrão de vida decente, avaliado pelo ganho per capita com a paridade em dólares americanos ${ }^{31}$. O Município de São Carlos obteve em 2004 um IDH de 0,841, a $67^{\mathrm{a}}$ posição entre as 5.507 cidades brasileiras ${ }^{32}$. Estudos prévios mostram que as mulheres que vivem em áreas de melhor desenvolvimento sócio-econômico têm os casos de câncer de mama diagnosticados em estadios mais iniciais, e isto apóia a hipótese de que os percentuais singulares de diagnósticos em estadio inicial e a proeminente posição sócio-econômica do município de São Carlos estejam inter-relacionados.

Este estudo é essencialmente descritivo e com uma amostra relativamente pequena, mas é baseado em uma série de casos de câncer de mama de uma população bem definida. Embora o percentual de ca- 
sos em estadio inicial seja comparável aos observados em registros populacionais de países cujos programas de detecção precoce do câncer de mama estão estabelecidos há muito mais tempo, há a indicação de que modificações em nossas campanhas devem ser feitas com o intuito de melhores taxas de detecção de tumores subclínicos, e assim o tratamento terá maior sucesso levando a melhor sobrevida ou cura.
Conclui-se que os dados do SM São Carlos provêem informações importantes sobre o câncer de mama em São Carlos, podem ser utilizados no direcionamento de ações de saúde que favoreçam o diagnóstico das mulheres com câncer de mama em um estadio cada vez mais inicial, e servem como referencial no acompanhamento da efetividade dos programas implantados.

Macchetti $\mathrm{AH}$. The staging of breast cancer diagnosed in São Carlos Public Health System. Medicina. (Rbeirão Preto) 2007; 40 (3): 394-402, july/sept.

ABSTRACT: Introduction: data on breast cancer stage at diagnosis are easily available for most developed countries while they are more difficult to obtain for countries of Latin America. Data of Instituto Nacional do Câncer reffer to $60 \%$ of breast cancer cases in late stage, however the present of Mastology Service of Heath Secretary of São Carlos showed different data that estimulate the evaluation of the breast cancer stage and age at diagnosis in the patients of public health system resident at São Carlos and comparation to statistics of others institutions. Patients and Methods: records of 106 women newly diagnosed with breast cancer between March 2000 and December 2004 at Mastology Service of Health Secretary of São Carlos (SUS) were reviewed. The breast cancer stage and age at diagnosis were annotade. Breast cancer stage was grouped according to TNM (AJCC) and SEER Summary Staging systems, and age at diagnosis was grouped according age under or above the median of the group of patients to correlate with the breast cancer stage. Results: In the TNM staging system the tumors in situ corresponded to $6,6 \%$ of all cases, tumors at stage I to $25,4 \%$, stage II to $44,3 \%$, stage III to $17,0 \%$ and stage IV to $6,6 \%$. In the SEER staging system the tumors in situ corresponded to $6,6 \%$ of all cases, local tumors to $62,2 \%$, regional tumors to $24,5 \%$ and diseminated tumors to $6,6 \%$. There were more early stage cases while using TNM $(76,3 \%)$ than while using SEER staging system $(68,8 \%)$. There was association of age group under 56 years with early breast cancer stage at diagnosis in AJCC staging system, but not in SEER staging system. Conclusion: relevant data to a better understanding of the breast cancer care are presented, permiting improve community-level interventions to cancer detection, and also a data-driven rationale for allocating resources to cancer-related policies, programs, and services.

Keywords: Breast Neoplasms. Neoplasm Staging. Health Services Epidemiology.

\section{REFERÊNCIAS}

1 - Instituto Nacional do Câncer. Estimativa 2006: Incidência do câncer no Brasil. Rio de Janeiro: INCA; 2005.

2 - Day NE, Williams DR, Khaw KT. Breast cancer screening programs: the development of a monitoring and evaluation system. Br J Cancer 1989;59(6):954-8.

3 - Marchick J, Henson DE. Correlations between access to mammography and breast cancer stage at diagnosis. Cancer 2005;103(8):1571-80.

4 - Rosenberg J, Chia YL, Plevritis S. The effect of age, race, tumor size, tumor grade, and disease stage on invasive duc- tal breast cancer survival in the U.S. SEER database. Breast Cancer Res Treat 2005;89(1):47-54.

5 - Bosetti C, Malvezzi M, Chatenoud L, Negri E, Levi F, La Vecchia C. Trends in cancer mortality in the Americas, 1970-2000. Ann Oncol 2005;16 (3): 489-511.

6 - Parkin DM, Bray F, Ferlay J, Pisani P. Estimating the world cancer burden: Globocan 2000. Int J Cancer 2001; 94 (2): 153-6.

7 - Schwartsmann G. Breast cancer in South America: challenges to improve early detection and medical management of a public health problem. J Clin Oncol 2001;19 (Suppl 18): S118S-24. 
8 - Bradley CJ, Given CW, Roberts C. Race, socioeconomic status, and breast cancer treatment and survival. J Natl Cancer Inst 2002;94(7):490-6.

9 - Lannin DR, Mathews HF, Mitchell J, Swanson MS, Swanson $\mathrm{FH}$, Edwards MS. Influence of socioeconomic and cultural factors on racial differences in late-stage presentation of breast cancer. JAMA 1998;279(22):1801-7.

10 - Richardson LC, Schulman J, Sever LE, Lee NC, Coate RJ. Early-stage breast cancer treatment among medically underserved women diagnosed in a national screening program, 1992-1995. Breast Cancer Res Treat 2001;69(2):13342.

11 - Liu MJ, Hawk H, Gershman ST, Smith SM, Karacek R, Woodford ML, Ayanian JZ. The effects of a National Breast and Cervical Cancer Early Detection Program on social disparities in breast cancer diagnosis and treatment in Massachusetts. Cancer Causes Control 2005;16(1):27-33.

12 - Roetzheim RG, Pal N, Tennant C, Voti L, Ayanian JZ, Schwabe $A$, Krischer JP. Effects of health insurance and race on early detection of cancer. J Natl Cancer Inst 1999; 91 (16): 1409-15.

13 - Oluwole SF, Ali AO, Adu A, Blane BP, Barlow B, Oropeza R, Freeman HP. Impact of a cancer screening program on breast cancer stage at diagnosis in a medically underserved urban community. J Am Coll Surg 2003;196(2):180-8.

14 - Bradley CJ, Given CW, Roberts C. Disparities in cancer diagnosis and survival. Cancer 2001;91(1):178-88.

15 - Singletary SE, Allred C, Ashley P, Bassett LW, Berry D, Bland KI, Borgen PI, Clark G, Edge SB, Hayes DF, Hughes LL, Hutter RV, Morrow M, Page DL, Recht A, Theriault RL, Thor A, Weaver DL, Wieand HS, Greene FL. Revision of the American Joint Committee on Cancer Staging System for Breast Cancer. J Clin Oncol 2002;20(17):3628-36.

16 - Roffers SD, Ries LAG, Fritz AG, Hurlbut AA (eds). SEER Summary Staging Manual - 2000: Codes and Coding Instructions. Bethesda, MD: National Cancer Institute; 2001.

17 - Vieira RAC, Heikel RL, Wohnrath DR, Hidalgo GS, Gomes UA. Aspectos epidemiológicos das 5001 pacientes tratadas por câncer de mama no Hospital de Câncer de Barretos [resumos] XIII Congresso Brasileiro de Mastologia. Rio de Janeiro; 2005. p. 46-7.

18 - Santos MCA, Passos MP, Sanches FB, Fuschino CMH, Filho JAR. Perfil das pacientes portadoras de câncer de mama operadas na Unidade de Mastologia do HBDF em 2002 [resumos]. XIII Congresso Brasileiro de Mastologia. Rio de Janeiro; 2005. p. 158-9.

19 - Michigan Department of Community Health. Community Cancer Incidence and Mortality. Stage at Diagnosis, 1985-2002. http://www.mdch.state.mi.us/pha/osr/Cancer/brsstg.asp. Acesso em 10 de Outubro de 2006.
20 - Minnesota Cancer Surveillance System. Cancer in Minnesota 1988-2002. http://www.health.state.mn.us/divs/hpcd/ cdee/mcss/documents/2005chapter3_5.pdf. Acesso em 10 de Outubro de 2006.

21 - Connecticut Tumor Registry. http://www.dph.state.ct.us/ OPPE/pdfs/ctrbook4-02issue.pdf. Acesso em 10 de Outubro de 2006.

22 - Oregon Partnership for Cancer Control. http:// www.oregon.gov/DHS/ph/cancer/prevention/breast. Acesso em 10 de Outubro de 2006.

23 - Instituto Nacional do Câncer. Registro hospitalar de câncer: dados dos hospitais do INCA. Relatório anual 1994/1998. Rio de Janeiro: INCA; 2004.

24 - Wunsch Filho V, Moncau JE. Cancer mortality in Brazil 19801995: regional patterns and time trends. Rev Assoc Med Bras 2002;48(3):250-7.

25 - Coburn NG, Chung MA, Fulton J, Cady B. Decreased breast cancer tumor size, stage, and mortality in Rhode Island: an example of a well-screened population. Cancer Control 2004;11(4):222-30.

26 - Hillner BE, Penberthy L, Desch CE, McDonald MK, Smith TJ, Retchin SM. Variation in staging and treatment of local and regional breast cancer in the elderly. Breast Cancer Res Treat 1996;40(1):75-86.

27 - Randolph WM, Goodwin JS, Mahnken JD, Freeman JL. Regular mammography use is associated with elimination of agerelated disparities in size and stage of breast cancer at diagnosis. Ann Intern Med 2002;137(10):783-90.

28 - Fundação Oncocentro de São Paulo (FOSP). Dados de câncer. http://www.fosp.saude.sp.gov.br/html/fr_mapa.html. Acesso em 10 de Outubro de 2006

29 - Instituto Brasileiro de Geografia e Estatística. Assistência Médica Sanitária 2002. Malha municipal digital do Brasil: situação em 2001. Rio de Janeiro: IBGE; 2005.

30 - Instituto Brasileiro de Geografia e Estatística. Resultados da Amostra do Censo Demográfico 2000 - Malha municipal digital do Brasil: situação em 2001. Rio de Janeiro: IBGE; 2004.

31 - Human Development Index. Human Development Report Office. http://hdr.undp.org/statistics/indices/about_hdi.cfm. Acesso em 10 de Outubro de 2006.

32 - Programa das Nações Unidas para o Desenvolvimento. Tabelas de ranking do IDH-M. http://www.pnud.org.br/atlas/ tabelas/index.php. Acesso em 10 de Outubro de 2006.

Recebido em 21/06/2006

Aprovado em 15/06/2007 